\title{
Enhancement of tumor cell death by combining cisplatin with an oncolytic adenovirus carrying MDA-7/IL-24
}

\author{
Yu-mei $\mathrm{WU}^{1}$, Kang-jian ZHANG ${ }^{1}$, Xue-tian $\mathrm{YUE}^{1}$, Yi-qiang WANG ${ }^{2}$, Yi YANG ${ }^{1}$, Gong-chu $\mathrm{LI}^{1}$, Na $\mathrm{LI}^{1}$, Yi-gang WANG ${ }^{1, *}$ \\ ${ }^{1}$ Xinyuan Institute of Medicine and Biotechnology, College of Life Science, Zhejiang Sci-Tech University, Hangzhou 310018, China; ${ }^{2}$ Department of Surgery, \\ Duchang People's Hospital, Jiujiang 332600, China
}

\begin{abstract}
Aim: The aim of this study was to creatively implement a novel chemo-gene-virotherapeutic strategy and further strengthen the antitumor effect in cancer cells by the combined use of ZD55-IL-24 and cisplatin.

Methods: ZD55-IL-24 is an oncolytic adenovirus that harbors interleukin 24 (IL-24), which has a strong antitumor effect and was identified and evaluated by PCR, RT-PCR, and Western blot analysis. Enhancement of cancer cell death using a combination of ZD55-IL-24 and cisplatin was assessed in several cancer cell lines by the 3-(4,5-dimethylthiazol-2-yl)-2,5-diphenyltetrazolium bromide (MTT) assay and cytopathic effect (CPE) assay. Apoptosis induction by treatment with ZD55-IL-24 and/or cisplatin was detected in BEL7404 and SMMC7721 by morphological evaluation, apoptotic cell staining, and flow cytometry analysis. In addition, negative effects on normal cells were evaluated in the L-02 cell line using the MTT assay, the CPE assay, morphological evaluation, apoptotic cell staining, and flow cytometry analysis.

Results: The combination of ZD55-IL-24 and cisplatin, which is superior to ZD55-IL-24, cisplatin, and ZD55-EGFP, as well as ZD55-EGFP plus cisplatin, resulted in a significantly increased effect. Most importantly, conjugation of ZD55-IL-24 with cisplatin had toxic effects equal to that of cisplatin and did not have overlapping toxicities in normal cells.

Conclusions: This study showed that ZD55-IL-24 conjugated with cisplatin exhibited a remarkably increased cytotoxic and apoptosis-inducing effect in cancer cells and significantly reduced the toxicity in normal cells through the use of a reduced dose.
\end{abstract}

Keywords: cisplatin; MDA-7/IL-24; oncolytic adenovirus; apoptosis

Acta Pharmacologica Sinica (2009) 30: 467-477; doi: 10.1038/aps.2009.16; published online 9th March 2009

\section{Introduction}

MDA-7/IL-24 (melanoma differentiation associated gene-7/interleukin-24), which was first classified as a member of the interleukin (IL)-10 gene family, has attracted particular attention ${ }^{[1]}$ because it can specifically induce apoptosis in a wide variety of malignant cells but spares normal cells ${ }^{[2,3]}$. Many studies proved that IL-24 exhibited potent "bystander antitumor" activity ${ }^{[4]}$ and enhanced radiation lethality ${ }^{[5]}$, induced immune-regulatory activities ${ }^{[6]}$, and inhibited tumor angiogenesis ${ }^{[7]}$. In addition, Ad.IL-24, which is a recombinant adenovirus that encodes the human IL-24 gene, has been evaluated in a phase I/II clinical trial in patients with advanced carcinoma ${ }^{[8,9]}$, which showed that

* Correspondence to Dr Yi-gang WANG.

E-mail wangyigang43@163.com

Received 2008-10-21 Accepted 2009-01-31
IL-24 was a potent therapeutic gene for human cancers. Recently, the novel oncolytic adenovirus vector, ZD55, was constructed by deleting an E1B 55-kDa gene of adenovirus 5. ZD55 is similar to the virotherapy agent, ONYX-015, which is an oncolytic adenovirus with a deleted E1B 55-KDa that selectively replicates in p53-deficient tumor cells and lyses them. The novel gene-virotherapy strategy for cancer using the delivery of therapeutic genes by ZD55 was first reported by us and indicated a strong antitumor effect both in vitro and in vivo ${ }^{[10,11]}$. We have previously demonstrated that ZD55-IL-24 exhibits a strong tumor suppression effect in a panel of tumor cells and has a high antitumor effect for human colorectal carcinoma xenografts in nude mice ${ }^{[12]}$. Furthermore, ZD55-IL-24 has been exploited as an anticancer drug in China for its potent antitumor activity.

Chemotherapy is one of the most conventional therapeutic strategies for human cancers. Cisplatin, which is also 
named cis diamminedichloroplatinum (DDP), is deemed to be the "penicillin of cancer drugs" due to its universal, early, and effective treatment for many cancers ${ }^{[13]}$. In fact, cisplatin is often used as part of an attractive chemotherapy regimen and is widely used to treat a variety of cancers, including ovarian, head and neck, bladder, prostate, cervical, testicular, lung, gullet, stomach, and other neoplasms. To date, the mechanism has not yet been fully elucidated, and cisplatin is generally believed to kill cancer cells by binding to DNA and interfering with the cell's repair mechanism, which eventually leads to cell death ${ }^{[14]}$. Unlike many anticancer drugs, which are organic molecules that have complex structures, cisplatin is an inorganic molecule with a simple structure. Despite these merits, severe toxic side effects and drug resistance are major clinical obstacles associated with cisplatin therapy ${ }^{[15,16]}$. The dose that is necessary to overcome even a small increase in cellular resistance can result in severe cytotoxicity in normal cells. Therefore, it is urgent to explore novel approaches to reduce drug dosage, minimize side effects, enhance the efficacy of therapy, and promote the application of cisplatin in cancer therapy.

Although either ZD55-IL-24 or cisplatin alone displays potent antitumor activity, some disadvantages exist, such as drug resistance and damage to normal cells. Thus, further investigation is required to increase the antitumor effects of these drugs. Chemo-gene-virotherapy, a novel strategy that combines a chemotherapeutic reagent, therapeutic genes, and an oncolytic virus (ZD55), was first proposed by us ${ }^{[17]}$. Previous reports have demonstrated that the combination of ZD55, carrying the TRAIL or Smac gene, and chemotherapeutic drugs significantly improved the tumor-killing effect and reduced side effects ${ }^{[17,18]}$.

In this study, we utilized the above strategy and combined cisplatin with ZD55-IL-24 to investigate antitumor efficacy. Our data proved that conjugation of cisplatin with ZD55IL-24 resulted in robust cytotoxicity in tumor cell lines without any overlapping toxicity in normal cells. In addition, negative effects were avoided by the use of the tumor specific-replication adenovirus and the decreased drug dose. This is the first study in which ZD55-IL-24 was applied in a novel chemo-gene-virotherapy strategy and it demonstrated the potential of gene therapy for human cancers.

\section{Materials and methods}

Cells and cell culture HEK293 (human embryonic kidney cell line containing the E1A region of adenovirus) was obtained from Microbix Biosystems, Inc (Toronto, Ontario, Canada). L-02 (normal human liver cell line),
BEL7404 (human hepatocellular carcinoma cell line, HCC), SMMC7721 (HCC cell line), H1299 (human lung adenocarcinoma cell line), HCT116 (human colorectal cancer cell line), HeLa (human cervical cancer cell line), and CNE (human nasopharyngeal carcinoma cell line) were purchased from the Shanghai Cell Collection (Shanghai, China). The HEK293 cell line was cultured in Dulbecco's modified Eagle's medium (DMEM; GIBCOBRL, Grand Island, NY) supplemented with $10 \%$ heat-inactivated fetal bovine serum (FBS; GIBCOBRL). Other cell lines were cultured in DMEM supplemented with $5 \%$ heat-inactivated FBS. All cell lines were cultured at $37^{\circ} \mathrm{C}$ in a $5 \% \mathrm{CO}_{2}$ humidified incubator.

Plasmids and generation of recombinant adenovirus The plasmids, pZD55-EGFP and pZD55-IL-24, were previously constructed in our laboratory. The oncolytic adenoviruses, ZD55-EGFP and ZD55-IL-24, were generated in HEK293 cells by homologous recombination between pZD55-EGFP or pZD55-IL-24 and the adenovirus packaging plasmid, pBHGE3 (Microbix Biosystems, Toronto, Canada), respectively ${ }^{[12]}$. ZD55-EGFP and ZD55-IL-24 were amplified in HEK293 cells, purified by cesium chloride gradient ultracentrifugation and subjected to dialysis. Virus titer was measured using a standard plaque formation assay in HEK293 cells.

Identification of recombinant viruses by conventional PCR The viral genome was extracted from the purified stock of ZD55-EGFP or ZD55-IL-24 using a QIAamp DNA blood mini kit (QIAGEN, Germany). The EGFP gene and IL-24 gene were verified by conventional PCR using the following primers (EGFP gene forward: 5'-AGCTGGACGGCGACGTAAAC-3' and reverse: 5'-CACGAACTCCAGCAGGACATG-3'; IL-24 gene forward: 5'-GAATTCGATATCTCTAGA C-3' and reverse: 5'-ATAGATATCTCAGAGCTTGTA-3'). The cycle conditions were $94^{\circ} \mathrm{C}$ for $5 \mathrm{~min}$, followed by 30 cycles at $94^{\circ} \mathrm{C}$ for $1 \mathrm{~min}, 50^{\circ} \mathrm{C}$ for $30 \mathrm{~s}, 72^{\circ} \mathrm{C}$ for $1 \mathrm{~min}$, and a final extension at $72{ }^{\circ} \mathrm{C}$ for $10 \mathrm{~min}$. The amplification product was visualized by electrophoresis on a $1 \%$ agarose gel containing ethidium bromide.

RT-PCR analysis Total cellular RNA was isolated using TRIzol reagent (Life Technologies, USA) according to the manufacturer's protocol. Reverse-transcription (RT)-PCR was performed with total RNA $(2 \mu \mathrm{g})$ using the First Strand RT-PCR Kit (Stratagene, USA). A cDNA equivalent of $1 \mathrm{ng}$ RNA was amplified by PCR using primers specific for the target gene. The forward and reverse primers for the IL-24 gene (649 bp) were 5'-GAATTCGATATCTCTAGAC-3' and 5'-ATAGATATCTCAGAGCTTGTA-3', respectively. The cycle conditions were the same as above. The amplified 
products were visualized by electrophoresis on a $1 \%$ agarose gel containing ethidium bromide. In addition, a control PCR for the detection of DNA contamination was performed using RNA samples devoid of the RT enzyme. The results were negative in all RNA samples, which indicated that our RNA preparations were not contaminated by viral DNA.

Western blot analysis Cells were harvested from the plates and resuspended in lysis buffer. Protein concentrations were determined with the Bio-Rad protein assay system. Total proteins were separated by sodium dodecyl sulfate-polyacrylamide gel electrophoresis (SDS-PAGE) on an $8 \%-12 \%$ gel and then transferred to a $0.45-\mu \mathrm{m}$ nitrocellulose membrane (Millipore, USA). The membrane was blocked with blocking buffer ( $5 \%$ bovine serum albumin, $10 \mathrm{mmol} / \mathrm{L}$ Tris- $\mathrm{HCl}, \mathrm{pH} 8.0,150 \mathrm{mmol} / \mathrm{L} \mathrm{NaCl}$, and $0.05 \%$ Tween 20 ) overnight at $4{ }^{\circ} \mathrm{C}$, and then incubated with primary antibodies (1:1000 dilutions). After incubation in the dark with IR Dye 700 or IR Dye 800 conjugated IgG secondary antibodies (1:4000 dilution, Rockland Inc, UK), immunodetection was performed using the Odyssey Infrared Imaging System (LICOR Biosciences, USA). The primary antibodies of actin, E1A, and poly (ADP-ribose) polymerase (PARP) were purchased from Santa Cruz Biotechnology (Santa Cruz, USA). The IL-24 primary antibody was obtained from GenHunter (Nashville, TN).

Cell viability assay Cells were plated in a 96-well plate and treated with the adenovirus, cisplatin (Alexis, Switzerland), or a combination of the virus and cisplation at the indicated MOI (Multiplicity of Infection, ratio of infectious virus particles to cells) or drug dosage. At the indicated time, the medium was removed and fresh medium containing 3-(4, 5-dimethylthiazol-2-yl)-2, 5-diphenyltetrazolium bromide (MTT, $0.5 \mathrm{mg} / \mathrm{mL}$, Sigma) was added to each well. Cells were incubated at $37{ }^{\circ} \mathrm{C}$ for $4 \mathrm{~h}$. The supernatant was removed, and $150 \mu \mathrm{L}$ DMSO was subsequently added to each well and mixed thoroughly. Absorbance was read at 595 $\mathrm{nm}$ with a Bio-Rad microplate reader (Hercules, CA).

Cytopathic effect (CPE) assay Human hepatocellular carcinoma cell lines (BEL7404 and SMMC7721), human cervical cancer cell line (HeLa), and normal liver cell line (L-02) were plated in a 96- or a 48-well plate and treated with cisplatin alone, the virus alone, or a combination of cisplatin and the virus at the indicated dosage. 72 or $120 \mathrm{~h}$ after treatment, the cells were washed, paraformaldehyde-fixed, and stained with crystal violet (Amresco, USA).

Flow cytometry analysis Cells were plated on 6-well plates at a certain density and treated with cisplatin alone, virus alone, or a combination of cisplatin and the virus at the indicated dosage. Cells were harvested $48 \mathrm{~h}$ after infec- tion, trypsinized, and washed once with complete medium. Aliquots of cells $\left(5 \times 10^{5}\right)$ were resuspended in $500 \mu \mathrm{L}$ of binding buffer and stained with fluorescein isothiocyanate (FITC)-labeled annexin V (BioVision, Palo Alto, CA). A fluorescence-activated cell-sorting (FACS; Becton Dickinson) assay was performed immediately after staining.

Morphological evaluation Cells were plated in a 48-well plate overnight and then treated with ZD55-EGFP and/or cisplatin or ZD55-IL-24 and/or cisplatin, and analyzed by microscopy for signs of apoptosis or cell death. The cells were examined $24 \mathrm{~h}$ and $48 \mathrm{~h}$ after treatment, respectively.

Apoptotic cell staining Cells seeded in 96-well plates were treated with ZD55-EGFP, ZD55-IL-24, cisplatin, ZD55-EGFP plus cisplatin, ZD55-IL-24 plus cisplatin, or phosphate-buffered saline (PBS). After $48 \mathrm{~h}$ or $72 \mathrm{~h}$ of treatment, the cells were incubated with Hoechst 33342 (Molecular Probes, Eugene, OR) for 30 min, washed with PBS twice, and observed under a fluorescence microscope.

Statistical analysis All data are displayed as means \pm SD. Student's $t$-test was applied to analyze the relationship between the different variables. Statistical significance was assumed when $P<0.05$.

\section{Results}

Characterization and Identification of recombinant oncolytic viruses The oncolytic adenovirus vector, ZD55, was constructed by deleting the $\mathrm{E} 1 \mathrm{~B} 55-\mathrm{kDa}$ gene of adenovirus 5 , which can selectively replicate and lyse in a large number of tumor cells. Based on the novel gene-virotherapy strategy, we utilized ZD55 to deliver the therapeutic gene IL-24 under the control of the human CMV-IE promoter and obtained the recombinant oncolytic adenovirus, ZD5-IL-24. The control virus, ZD55-EGFP, was constructed using the same methods. The packaging and purification of recombinant virus were performed as previously described $^{[21]}$, and the titer of ZD55-IL-24 and ZD55-EGFP contained $5 \times 10^{9}$ plaque-forming unit $(\mathrm{pfu}) / \mathrm{mL}$ and $1 \times 10^{10}$ $\mathrm{pfu} / \mathrm{mL}$, respectively. To identify the expected recombinant virus, the virus DNAs were extracted and PCR was performed to detect the corresponding exogenous gene (IL24 and EGFP) of the recombinant virus. There is a distinct DNA fragment from the IL-24 (649 bp) and EGFP (623 bp) genes in each virus (Figure 1A, 1B).

To assess the transgenic delivery capability that is mediated by oncolytic adenovirus, ZD55, the HCC cell line BEL7404 was infected with ZD55-IL-24 for $48 \mathrm{~h}$, and RT-PCR was performed to detect the expression of IL-24 

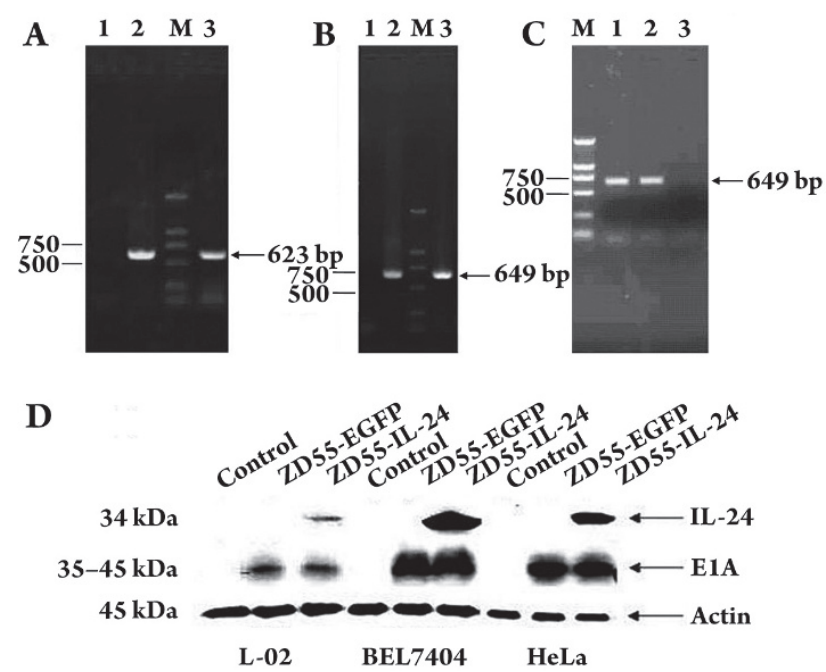

Figure 1. Identification of recombinant oncolytic adenovirus. (A) Identification of the IL-24 gene in ZD55-IL-24 by PCR. Lane 1: negative control; lane 2: positive control; lane M: DL2000 marker; lane 3: ZD55-IL-24. (B) Identification of EGFP in ZD55-EGFP by PCR. Lane 1: negative control; lane 2: positive control; lane M: DL2000 marker; lane 3: ZD55-EGFP. (C) The expression of IL-24 was detected by reverse transcription polymerase chain reaction (RT-PCR). Lane M: DL2000 marker; lane 1: pZD55-IL-24 (positive control); lane 2: ZD55-IL-24; lane 3: negative control. (D) Expression of E1A and IL-24 genes in L-02, BEL7404, and HeLa cells $48 \mathrm{~h}$ after infection of ZD55-EGFP.

gene. The results showed that $I L-24$ gene express efficiently in BEL7404 cells (Figure 1C). Furthermore, to prove the tumor-selective replication ability of the oncolytic adenovirus, we detected the expression of the adenovirus E1A protein and the therapeutic gene $I L-24$ by Western blot analysis in two tumor cell lines (BEL7404 and HeLa) and a normal cell line (L-02) that were infected with ZD55-EGFP and ZD55-IL-24. The strong expression of E1A in tumor cells indicated that ZD55-EGFP and ZD55-IL-24 could replicate at higher levels in tumor cells than in normal cells (Figure 1D). In addition, the expression of the $I L-24$ gene in tumor cells treated ZD55-IL-24 was observed at a much higher level than in normal cells. These results suggest that the oncolytic adenovirus ZD55 is tumor-specific and could significantly mediate efficient exogenous gene expression in tumor cells.

Enhanced antitumor effect using a combination of ZD55-IL-24 and cisplatin As a conventional chemotherapeutic drug, cisplatin was reported to augment IL-24 or ONYX-015-mediated virotherapy in tumor cells from several origins $^{[19,20]}$. To evaluate the cytotoxic effects of ZD55-IL-24 and cisplatin, two HCC cell lines (BEL7404, SMMC7721), a human lung cancer cell line (H1299), a human colorectal cancer cell line (HCT116), a human cervical cancer cell line (HeLa), and a human nasopharyngeal carcinoma cell line (CNE) were infected with ZD55-IL-24 and ZD55EGFP separately or in combination with cisplatin. The MTT assay was performed, and the results are shown in Figure 2 $(P<0.05)$. The cell viability in the six tumor cell lines treated with a combination of ZD55-IL-24 (5 MOI) and cisplatin $(3 \mu \mathrm{g} / \mathrm{mL})$ decreased to approximately $30 \%-80 \%$ in a timedependent manner compared with ZD55-EGFP (5 MOI) alone, ZD55-IL-24 (5 MOI) alone, cisplatin $(3 \mu \mathrm{g} / \mathrm{mL})$ alone, or ZD55-EGFP (5 MOI) plus cisplatin $(3 \mu \mathrm{g} / \mathrm{mL})$. About $81 \%$ of HeLa cells and $73 \%$ of SMMC 7721 cells were killed by the combination of ZD55-IL-24 and cisplatin for $72 \mathrm{~h}$, but such a phenomenon could not be observed with other treatments. In addition, ZD55-EGFP or ZD55-IL-24 in combination with cisplatin was significantly different from either treatment alone for the six kinds of tumor cells $(P<0.05)$, which indicated that the combination of the oncolytic adenovirus with cisplatin had an obviously enhanced antitumor effect. Other than CNE cells, comparison of the other five tumor cells with regard to treatments using ZD5-IL-24 plus cisplatin and ZD55-EGFP plus cisplatin was significant $(P<0.05)$ or very significant $(P<0.01)$, which indicates that the $I L-24$ gene can induce tumor cell apoptosis and kill the cells. The results suggest that the combination of ZD55-IL-24 and cisplatin has an enhanced tumor-killing effect.

The cytotoxic effects of ZD55-IL-24 or cisplatin and the combination treatment on HeLa, SMMC7721, and BEL7404 were also assessed by crystal violet staining (Figure 3). When the cells were treated with a combination of ZD55-IL-24 and cisplatin, the enhanced cytotoxicity was significantly more potent than that of the other treatments.

Apoptosis induction by treatment with ZD55-IL-24 and/or cisplatin ZD55-IL-24 could efficiently mediate IL-24 expression in tumor cells, as shown in our previous study ${ }^{[21]}$. We detected IL-24 protein expression in HCC BEL7404 cells and human cervical cancer HeLa cells by Western blotting (Figure 1D). To determine whether cisplatin affects IL-24 expression, we treated the cancer cell line, BEL7404, with ZD55-IL-24 alone or ZD55-IL-24 plus cisplatin. The results indicated that cisplatin did not attenuate the expression of IL-24 (Figure 4). To determine the underlying mechanism by which ZD55-IL-24, cisplatin alone, or a combination of ZD55-IL-24 and cisplatin can induce apoptosis in cancer cells, the activation of death substrate poly (ADPribose) polymerase (PARP) was detected by Western blot analysis. The results showed that the cleavage of PARP (p85, the active form of PARP, which is typical of apoptosis) was observed in the tumor cells, BEL7404 and HeLa, after infec- 

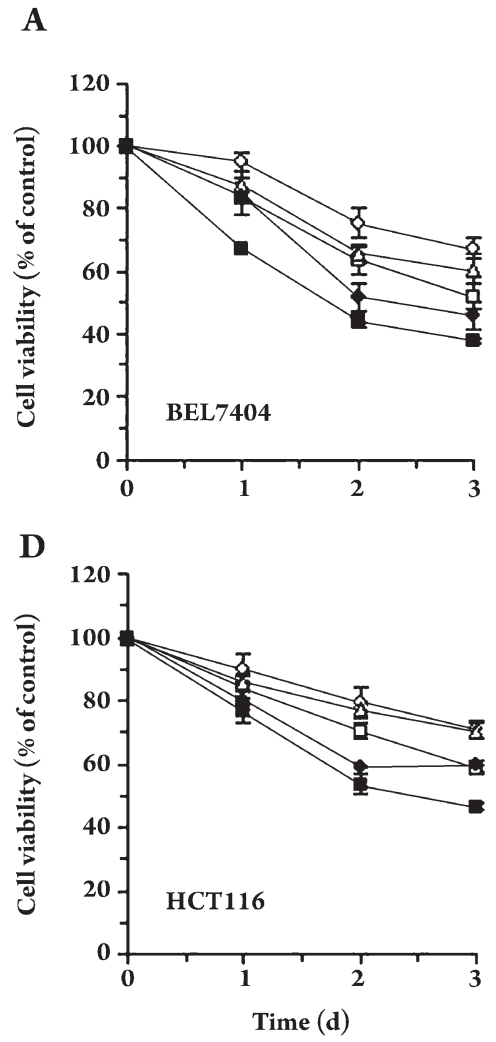

B

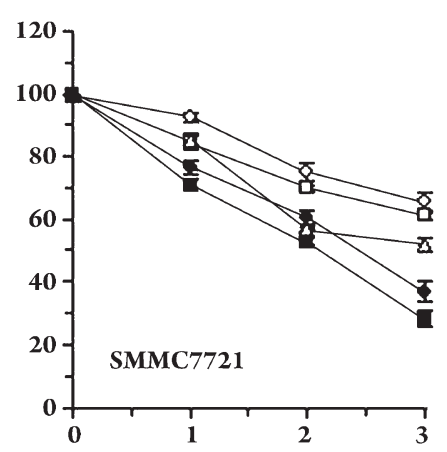

E

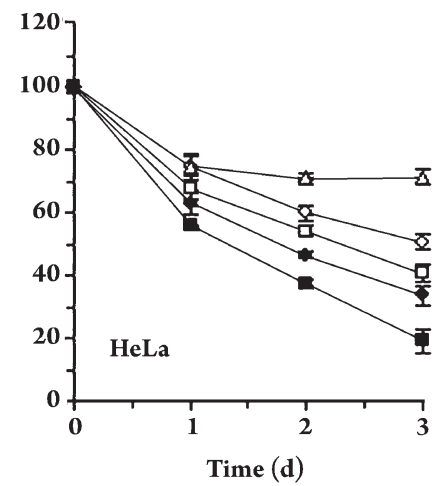

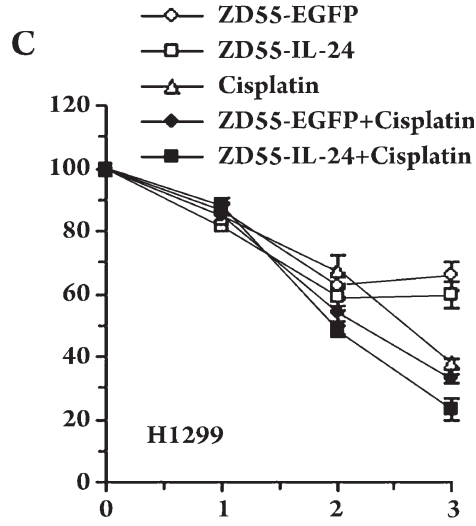

F

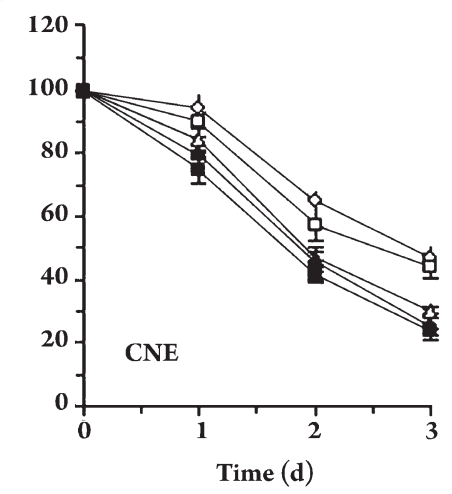

Figure 2. Enhanced suppression of tumor cell proliferation using the combination of ZD55-IL-24 and cisplatin. The tumor cells BEL7404 (A), SMMC7721 (B), H1299 (C), HCT116 (D), HeLa (E), and CNE (F) were treated with ZD55-EGFP (5 MOI), ZD55-IL-24 (5 MOI), cisplatin (3 $\mu \mathrm{g} / \mathrm{mL})$, ZD55-EGFP (5 MOI)+cisplatin $(3 \mu \mathrm{g} / \mathrm{mL})$, or ZD55-IL-24 (5 MOI)+cisplatin $(3 \mu \mathrm{g} / \mathrm{mL})$. Cell viability was determined by the MTT assay. Data are presented as means $\pm \mathrm{SD}$ of three independent experiments.

tion with ZD55-IL-24 for $48 \mathrm{~h}$ but not in the normal L-02 cells, which suggested that our oncolytic virus could cause tumor-specific apoptosis (Figure 5).

Flow cytometry analysis was performed to confirm the enhanced apoptosis of tumor cells after treatment with combined ZD55-IL-24 and cisplatin therapy. The percentage of apoptotic cells was determined by annexin $\mathrm{V}$ staining. The combination of ZD55-IL-24 with cisplatin showed a higher percentage of cell apoptosis in SMMC7721 and BEL7404 cells compared with other groups (Figure 6A). These observations were consistent with the morphological features. Most tumor cells died during treatment with combined ZD55-IL-24 and cisplatin, as shown by cell surface blebbing and the formation of apoptotic bodies (Figure 6B). Additionally, Hoechst 33342 staining also was performed to detect the apoptotic morphological changes of tumor cells after each treatment using a fluorescence microscope. More tumor cells treated with a combination of ZD55-IL-24 and cisplatin showed obvious apoptosis, including chromatin condensation and nuclear fragmentation (arrows indicate apoptotic cells), compared with other treatments (Figure 6B, $6 \mathrm{C})$.

Taken together, ZD55-IL-24 could lead to efficient tumor-specific viral replication and IL-24 expression and significantly induce tumor cell apoptosis. The combination of ZD55-IL-24 and cisplatin resulted in a significantly enhanced antitumor effect.

A dose-dependent manner of cytotoxicity in normal cells To evaluate the safety of the cisplatin and virus combination, it is necessary to use a proper dosage that not only kills cancer cells but also has significantly reduced side effects on normal cells. According to the MTT data $(P<0.05$; Figure $7 \mathrm{~A})$, the lowest drug dosage of cytotoxicity was determined to be 5 MOI of ZD55-IL-24 and $3 \mu \mathrm{g} / \mathrm{mL}$ of cisplatin. Subsequent $\mathrm{CPE}$ assays, morphological evaluation, and apoptotic cell staining further confirmed that this dose of $5 \mathrm{MOI}$ ZD55-IL-24 and $3 \mu \mathrm{g} / \mathrm{mL}$ cisplatin was reliable (Figure 7B, $7 \mathrm{C}, 7 \mathrm{D})$. As a tumor-selective replication vector that drives 


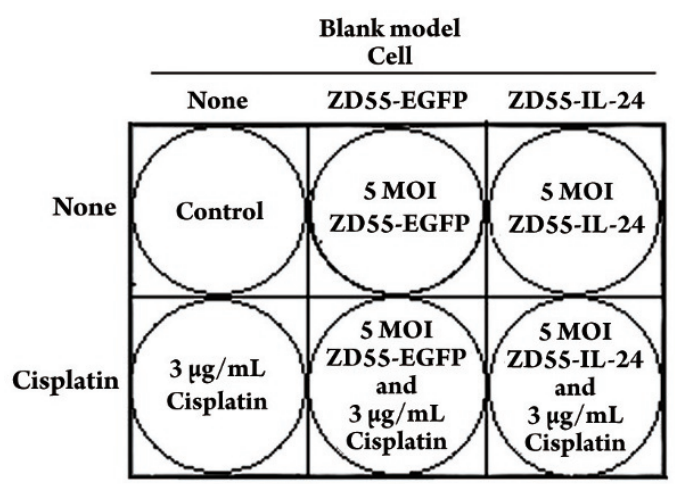

HeLa
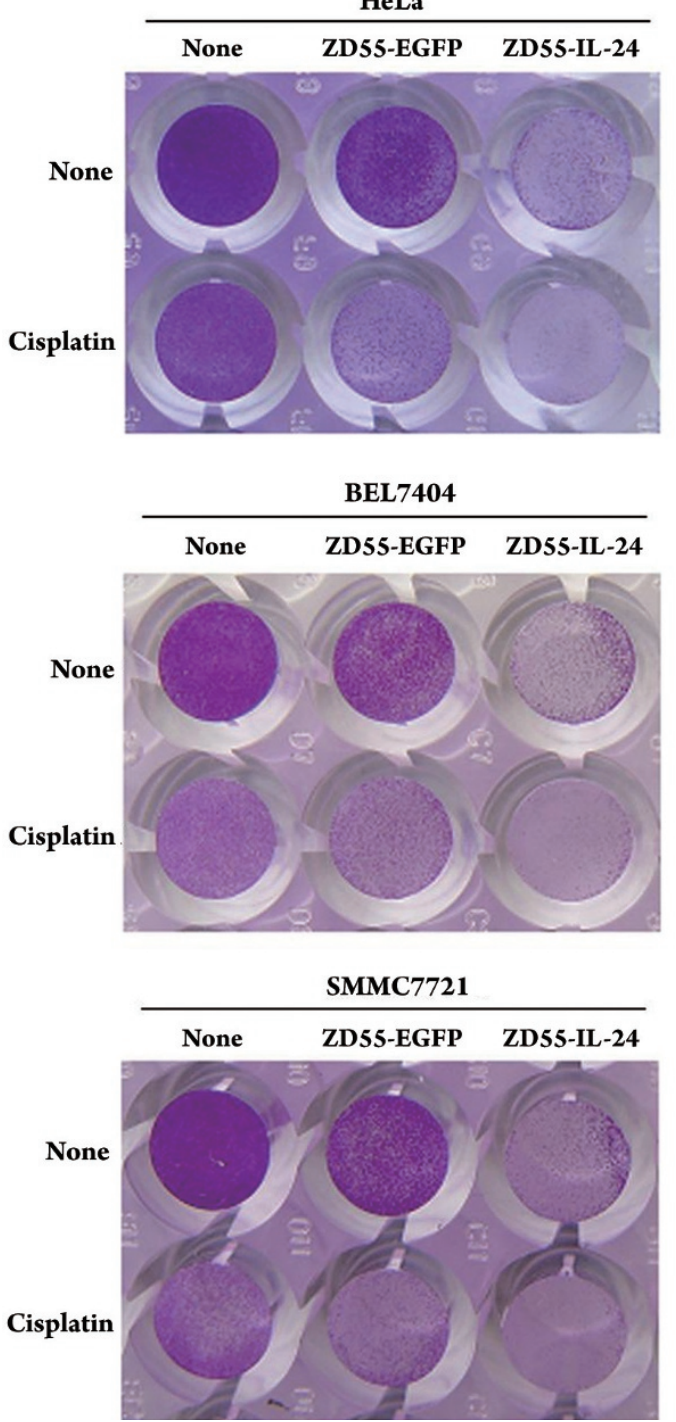

Figure 3. The $\mathrm{CPE}$ assay was performed by crystal violet staining. The tumor cell lines BEL7404, SMMC7721, and HeLa were infected with ZD55-EGFP, ZD55-IL-24, cisplatin, a combination of ZD55-EGFP and cisplatin, or a combination of ZD55-IL-24 and cisplatin at the indicated doses. $120 \mathrm{~h}$ later, the cells were stained with crystal violet.

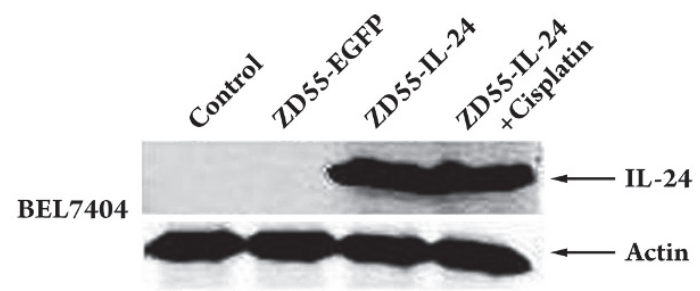

Figure 4. Effect of cisplatin on the expression of the $I L-24$ gene. IL24 was detected by Western blot analysis when BEL7404 cells were infected with ZD55-EGFP or ZD55-IL-24.

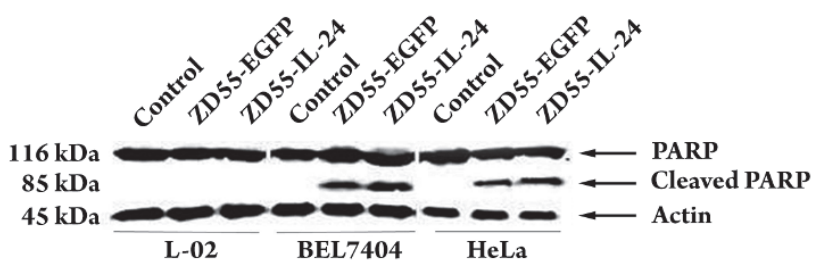

Figure 5. PARP activation in cells infected with ZD55-EGFP or ZD55-IL-24. Expression of PARP and its active form, p85 (cleaved PARP), was detected by Western blot analysis in L-02, BEL7404, and HeLa cells $48 \mathrm{~h}$ after infection with ZD55-EGFP or ZD55-IL-24.

a cancer specific pro-apoptotic gene, ZD55-IL-24 could theoretically spare normal cells. However, an extremely high dosage could still cause damage to normal cells (Figure 7A). Approximately $40 \%$ of L-02 cells were killed with ZD55IL-24 treatment at $80 \mathrm{MOI}$. The dose-dependent effect of cisplatin (Figure 7A) demonstrated that more than 70\% of L-02 cells were killed at the highest concentration (48 $\mu \mathrm{g} / \mathrm{mL}$ ) but only about $10 \%$ of normal cells died at the lowest concentration $(3 \mu \mathrm{g} / \mathrm{mL})$. Similarly, the combination of ZD55-IL-24 and cisplatin also resulted in dose-dependent cell death. Most importantly, the combination of ZD55IL-24 and cisplatin had approximatively equal toxic effects compared with cisplatin alone but did not have any overlapping toxicity in normal cells.

Morphological evaluation showed that most L-02 cells that were treated with ZD55-IL-24 (80 MOI) and cisplatin $(48 \mu \mathrm{g} / \mathrm{mL})$ were killed after $24 \mathrm{~h}$, a trend that became more obvious after $48 \mathrm{~h}$ (Figure 7C). However, the L-02 cells treated with ZD55-IL-24 (5 MOI) and cisplatin $(3 \mu \mathrm{g} / \mathrm{mL})$ exhibited no apparent apoptosis, similar to that observed in the CPE analysis (Figure 7B). In addition, apoptotic cell staining clearly indicated that higher doses resulted in remarkable chromatin condensation and nuclear fragmentation in L-02 cells (Figure 7D).

To validate whether the combinatorial treatment with 3 
A

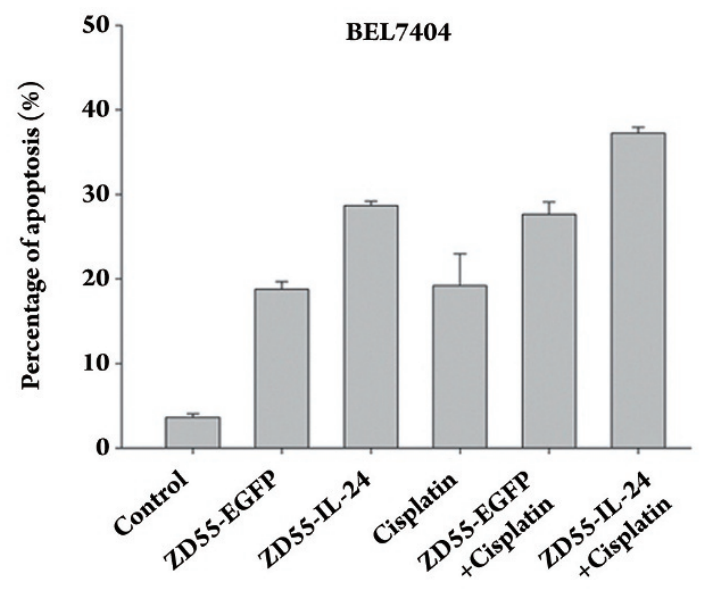

B

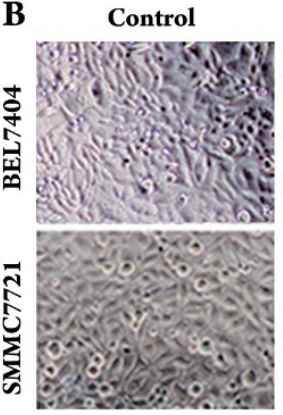

C
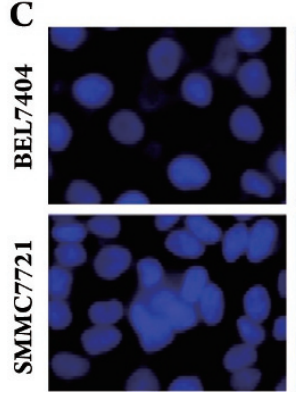

\section{ZD55-EGFP}
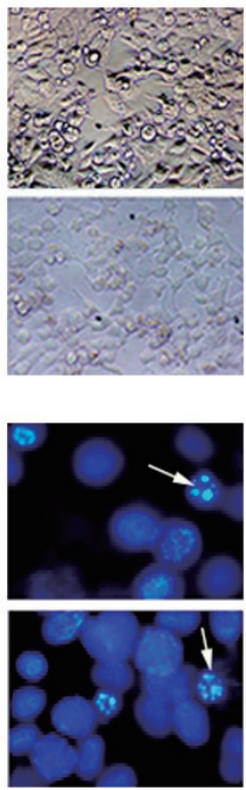

ZD55-IL-24
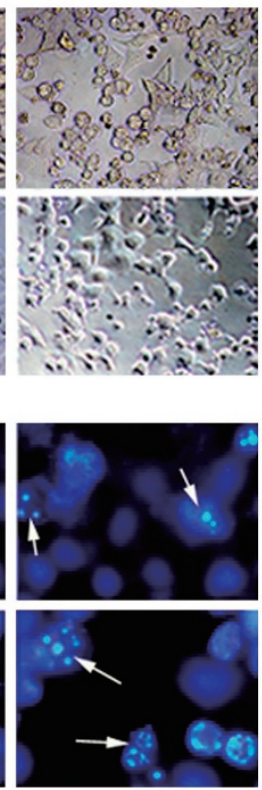
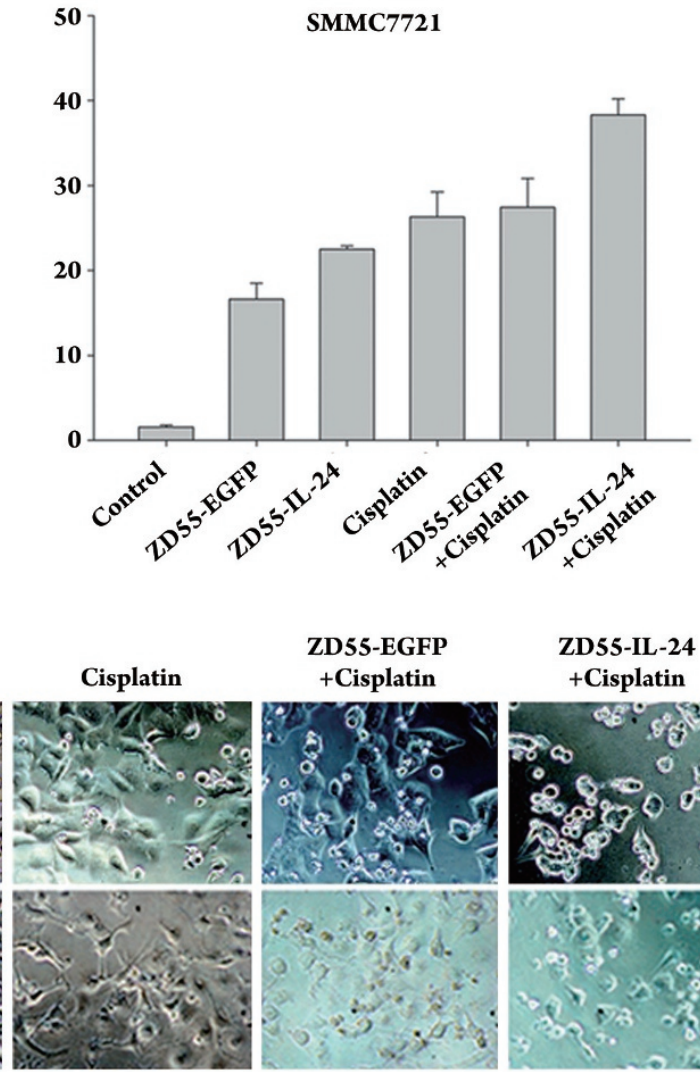

ZD55-IL-24

+Cisplatin
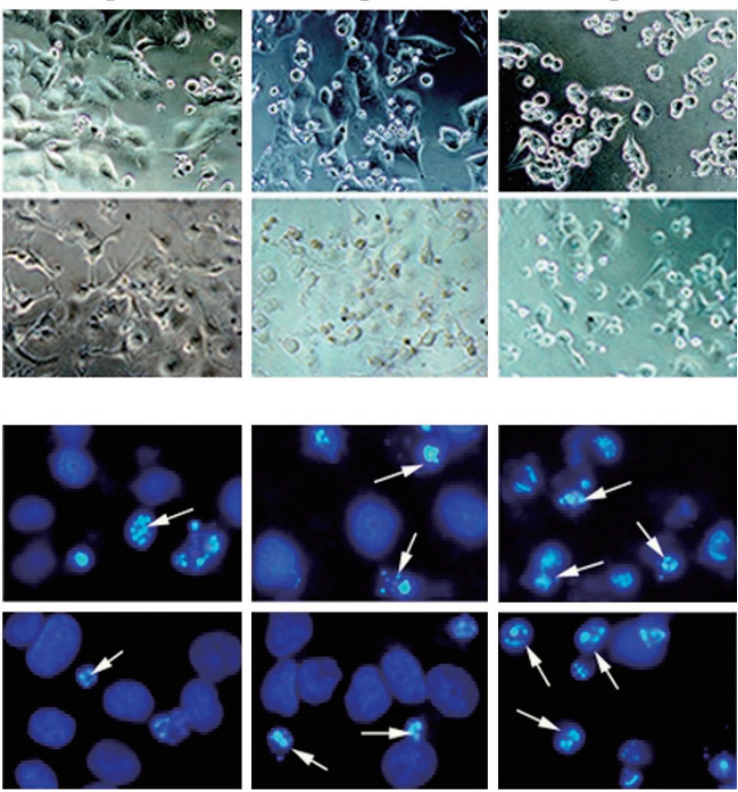

Figure 6. Detection of apoptosis and morphological evaluation of tumor cells. (A) Annexin V binding assay. The BEL7404 and SMMC7721 cells were treated with PBS, ZD55-EGFP, ZD55-IL-24, cisplatin, ZD55-EGFP plus cisplatin, or ZD55-IL-24 plus cisplatin at the indicated dose. $72 \mathrm{~h}$ later, the cells were harvested and stained with annexin V-FITC, which was immediately followed by flow cytometry analysis for apoptosis. The percentage of apoptotic cells was calculated using CellQuest software. Each value represents the mean of three wells. (B) BEL7404 cells and SMMC7721 cells were treated as above and the morphological changes of dead tumor cell or cells undergoing apoptosis were analyzed by microscopy after $72 \mathrm{~h}$ (Original magnification: $\times 200$ ). (C) Cells were incubated with Hoechst 33342 for 30 min. and condensation and fragmentation of nuclei were observed under a fluorescence microscope (arrow) (Hoechst 33342 stain; original magnification: $\times 200$ ).

$\mu \mathrm{g} / \mathrm{mL}$ cisplatin and 5 MOI ZD55-IL-24 affected the survival of normal cells, an MTT assay was performed to evaluate the cell viability. The survival of L-02 cells was seldom affected by the combinatorial treatment (Figure 8A). The toxic effect of the conjugation group is similar to that of cisplatin, which showed that it was safe to use in normal cells.
The annexin $\mathrm{V}$ binding assay also indicated that the combination of oncolytic virus and cisplatin did not lead to increased cell apoptosis compared with the individual treatments (Figure $8 \mathrm{~B}$ ). The results indicated that the above combination treatment was a potent anticancer therapy strategy and hardly affected the viability of normal cells. 

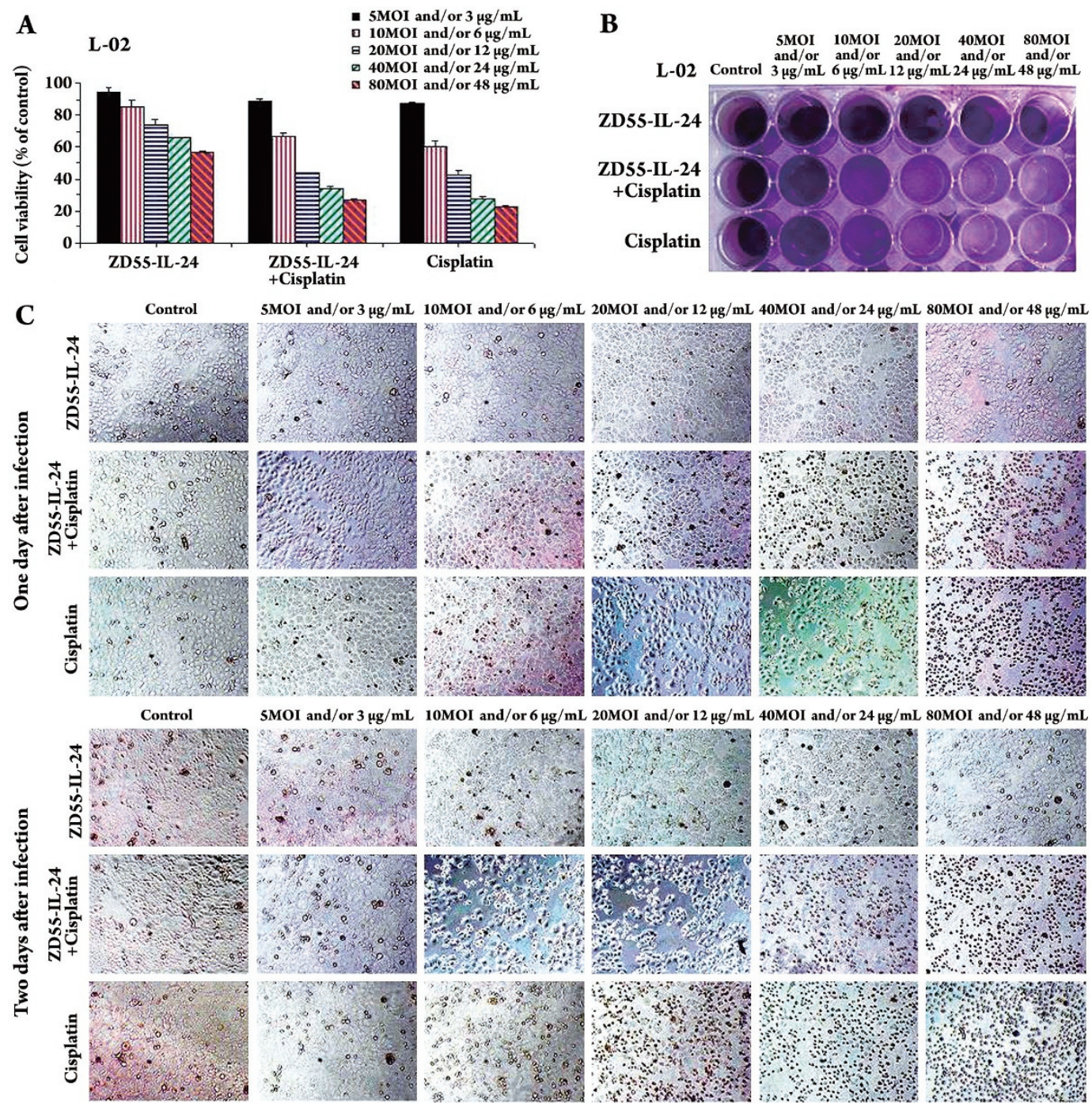

$5 M O I$ and/or $3 \mu \mathrm{g} / \mathrm{mL}$ 10MOI and $/$ or $6 \mu \mathrm{g} / \mathrm{mL} 20$ MOI and $/$ or $12 \mu \mathrm{g} / \mathrm{mL} 40 \mathrm{MOI}$ and $/$ or $24 \mu \mathrm{g} / \mathrm{mL} 80 \mathrm{MOI}$ and $/ \mathrm{or} 48 \mu \mathrm{g} / \mathrm{mL}$
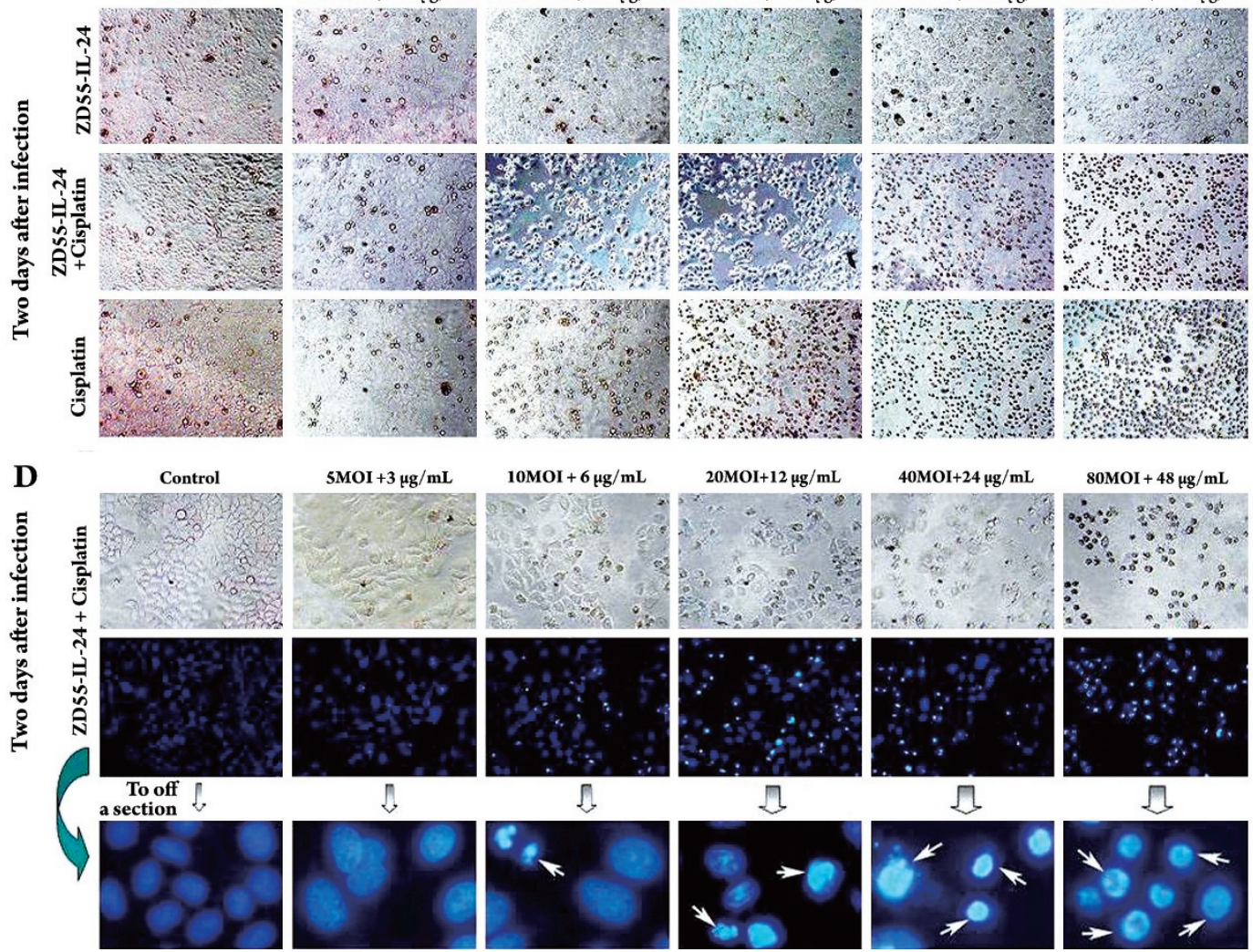

V.
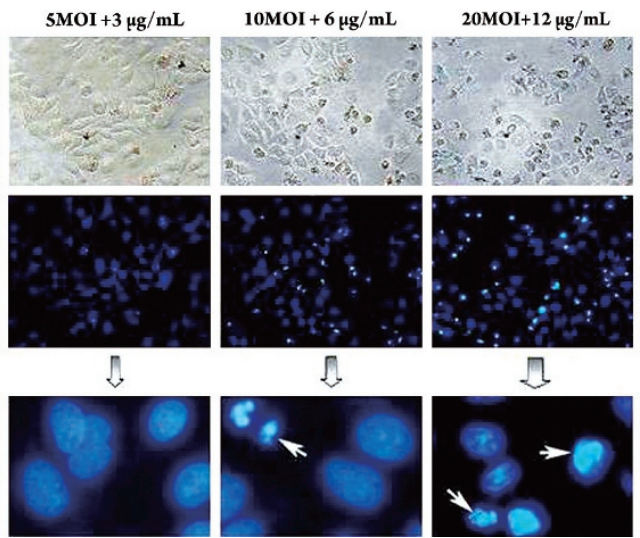

12

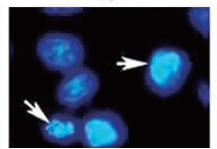

Figure 7. Dose-dependent manner of toxicity to normal cells. (A) The MTT assay was used to determine the viability of L-02 cells at various doses of ZD55-IL-24, ZD55-IL-24 + cisplatin, or cisplatin after $48 \mathrm{~h}$ of treatment. Data are presented as means \pm SD of three independent experiments. (B) CPE assay. Normal L-02 cells were treated with ZD55-IL-24, ZD55-IL-24+cisplatin, or cisplatin at the indicated doses. $72 \mathrm{~h}$ later, the cells were stained with crystal violet. (C) Morphological evaluation. L-02 cells were treated with ZD55-IL-24, ZD55-IL-24+cisplatin, or cisplatin and cell death was analyzed by microscopy after $24 \mathrm{~h}$ and $48 \mathrm{~h}$. (Original magnification: $\times 100$ ) (D) Apoptotic cell staining. Normal L-02 cells were treated with ZD55-IL-24 plus cisplatin at the indicated dose. Two days later, the cells were incubated with Hoechst 33342 and the condensation and fragmentation of nuclei was observed under a fluorescence microscope (arrow) (Hoechst 33342 stain; original magnification: $\times 200$ ). 

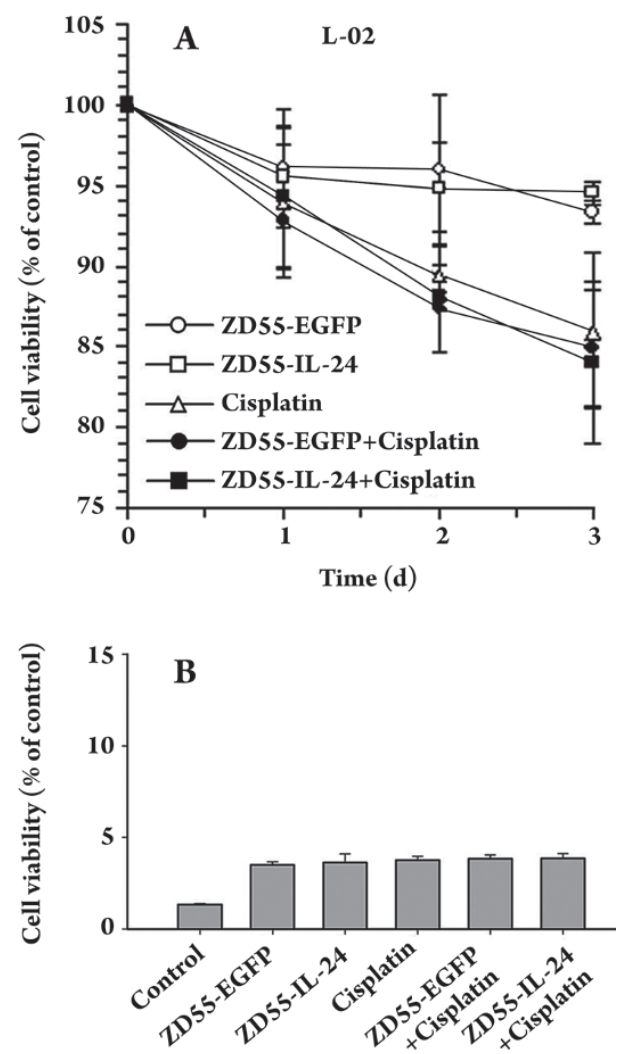

Figure 8. Combinatorial treatment with cisplatin and ZD55-IL-24 did not significantly affect the survival of normal cells. (A) L-02 cells were treated with ZD55-EGFP (5 MOI), ZD55-IL-24 (5 MOI), cisplatin $(3 \mu \mathrm{g} / \mathrm{mL})$, ZD55-EGFP (5 MOI)+cisplatin $(3 \mu \mathrm{g} / \mathrm{mL})$, or ZD55-IL$24(5 \mathrm{MOI})+$ cisplatin $(3 \mu \mathrm{g} / \mathrm{mL})$. Cell viability was determined by the MTT assay. Data are presented as means \pm SD of three independent experiments. (B) Apoptosis detection by annexin $\mathrm{V}$ binding assay. The L-02 cells were treated as in Figure 8A. $72 \mathrm{~h}$ later, the cells were harvested and stained with annexin V-FITC, which was immediately followed by flow cytometry. The percentage of apoptotic cells was calculated using CellQuest software. Each value represents the mean of 3 wells.

\section{Discussion}

MDA-7/IL-24 is a novel cancer growth-suppressing and apoptosis-inducing gene acquired by subtraction hybridization using a human melanoma cell line ${ }^{[1]}$. Some studies have indicated that increased expression of the IL-24 gene suppresses cell growth and induces cell apoptosis in a variety of cancer cells with single or multiple genetic defects, including alterations in $\mathrm{p} 53$, $\mathrm{p} 16 / \mathrm{INK} 4 \mathrm{a}$, and $\mathrm{Rb}^{[2,21]}$. The $I L-24$ gene is currently widely utilized in cancer gene therapy. Adenovirus mediated IL-24 gene overexpression in tumor cells is a promising way to improve cancer therapy. In our study, an E1B $55 \mathrm{kDa}$-deleted oncolytic adenovirus vector (ZD55) was engineered to specifically replicate and cause significant cytotoxic effects in all tested tumor cells while exhibiting low side effects in normal cells ${ }^{[10,22]}$. Using the gene-virotherapy strategy, the ZD55-mediated IL-24 gene (ZD55-IL-24) exhibited potent antitumor activity and may represent a novel approach to cancer therapy.

Cisplatin is an inorganic compound that is broadly used for the treatment of various forms of malignant tumors, in particular head and neck cancer and several pediatric malignancies. However, these so-called virtues are often offset by disadvantages, including chemoresistance and toxicity ${ }^{[23,24]}$. To eliminate drug resistance and reduce side effects, several conjugated strategies have been applied successfully in cancer therapy, such as the combination of cisplatin with the anticancer gene $I L-24$ or the viro-agent ONYX-015 $5^{[19,20]}$. Using these methods, potent antitumor effects and reduced side effects have been obtained with the combination of two regimens and a reduced dose. However, further improvements are still required to facilitate such strategies for clinical cancer therapy.

Previous studies in our laboratory have shown that ZD55-IL-24 could selectively replicate in the tumor cell lines Bcap37, BEL7404, HeLa, SMMC7721, HCT116, and SW620 and that overexpression of IL-24 suppressed tumor cell growth and induced apoptosis ${ }^{[12]}$. In this study, we used the chemo-gene-virotherapy strategy and comprehensively combined cisplatin with both IL-24 and ZD55. The combination of ZD55-IL-24 and cisplatin was first performed to treat a variety of tumor cells, and an empiric inhibition of tumor efficacy has been demonstrated. Western blot analysis has distinctly addressed the specific viral replication in BEL7404 and HeLa cells (Figure 1D). Notably, although ONYX-015 has been used extensively in cancer clinical trials, it has not been proven to be very efficient ${ }^{[25,26]}$. Nevertheless, ZD55-IL-24, which is an oncolytic adenovirus that harbors the therapeutic gene $I L-24$, cannot specifically replicate and efficiently express the $I L-24$ gene in cancer cells (Figure 1). However, it does lead to robust cancer-specific cytotoxicity (Figures 2, 3). Thus, this combinatorial strategy with ZD5-IL-24 and cisplatin has become an intriguing prospect for clinical cancer therapy.

The purpose of this study was to identify the enhanced cytotoxicity of cisplatin with gene-virotherapy agent ZD5-IL-24 in cancer cells and to evaluate the negative effects in normal cells. The MTT analysis showed that conjugation of cisplatin with ZD55-IL-24 was obviously superior to cisplatin, ZD55-EGFP, ZD55-IL-24 alone, or ZD55-EGFP plus cisplatin (Figure 2). However, a difference in cytotoxicity among the six tumor cells exists after various treatments. 
The difference may be caused by the sensitivity of different tumor cells to the adenovirus, the IL-24 gene, the chemotherapy drug, or their different cellular mechanisms. Some reports have indicated that the adenovirus-mediated IL-24 gene can be significantly expressed in tumor cells and lead to the specific apoptosis of tumor cells ${ }^{[6,9,12]}$. IL-24 triggers the external pathway of cell apoptosis, such as the activation of the caspase family, including the cleavage of caspase 3, 9, and PARP. The intrinsic pathway of apoptosis involves mitochondria cytochrome $c$ release. In addition, IL-24 can improve antitumor immunity and suppress tumor angiogenesis. It indicated that oncolytic adenovirus mediated IL-24 induced a strong cleavage of PARP by Western blot analysis (Figure 5). Hoechst33342 staining indicated that extensive chromatin condensation and nuclear fragmentation, which is a typical sign of cell apoptosis, was observed in the combined treatment of cisplatin with ZD55-IL-24 (Figure 6). However, the results have also shown that ZD55-EGFP exhibited a small antitumor effect and led to the apoptosis of tumor cells through the small cleavage of PARP compared with the ZD55-IL-24 plasmid (Figure 5). This suggests that the IL-24 gene has a significant role in tumor cell apoptosis. Oncolytic adenovirus can proliferate in tumor cells, lyse them, and achieve a vigorous tumor-killing effect ${ }^{[10,25,26]}$. Furthermore, some adenovirus structure proteins, such as E1A and E4, have an affect on the induction of tumor cell apoptosis.

ZD55-IL-24 had a dual role in both gene therapy and virotherapy, which led to a further improvement in the antitumor effect when it was combined with cisplatin. The mechanism of combined therapy includes an increased tumor killing effect, the promotion of tumor cell apoptosis, and reduced side effects in normal cells (Figures 2, 6, 7). The conjugation of ZD55-IL-24 and cisplatin may cause cell cycle arrest in tumors, stimulate an antitumor immune response, and exhibit a "bystander effect" to inhibit tumor angiogenesis and vascular epithelial growth factor. ZD55-IL-24 may also improve tumor cell sensitivity to cisplatin and reduce the resistance of cancer cells to cisplatin. The strong antitumor mechanism of this combination is still unclear and requires further investigation.

The severe side effects of high-dose chemotherapy with cisplatin are the essential obstacles in the treatment of solid tumors ${ }^{[27,28]}$. With cisplatin and ZD55-IL-24 working together, the dose could be expected to not only dramatically enhance the tumor killing effect but also significantly reduce the toxicity in normal cells. The MTT assay in L-02 normal cells led us to determine the minimal dose (5 MOI ZD55IL- $24+3 \mu \mathrm{g} / \mathrm{mL}$ cisplatin) in the current test. The cytotoxicity assay, morphological evaluation, and apoptotic detection showed very slight damage to normal cells at the minimal dose (Figures 7, 8).

This study is the first to show the combined use of ZD55IL-24 and cisplatin in cancer therapy. Conjugation of ZD55IL-24 with cisplatin could cause remarkable cytotoxicity in several cancer cell lines and significantly abolish side effects in normal cells through a reduction in the required dose. In addition, we have demonstrated that the current chemogene-virotherapy (cisplatin+ZD55-IL-24) strategy is superior to the conventional chemo-gene (cisplatin+IL-24) or chemo-viro (cisplatin+ONYX-015) approach. We therefore conclude that the chemo-gene-virotherapy, with a combination of ZD55-IL-24 and cisplatin, may be a powerful and effective strategy for clinical cancer treatment.

\section{Acknowledgments}

We thank Prof Xin-yuan LIU for his thorough review and approval of this article and Kan CHEN for her help with cell culture. This work was supported by the Zhejiang Science and Technology Support Plan (№ 2007C33027), National Natural Science Foundation of China (№ 30800093, № 30801379), Science and Technology Commission of Shanghai Municipality (№ 06DZ22032), National Basic Research Program of China (973 Program, № 2004 CB51804), Key Project of the Chinese Academy of Science (No KSCX2YW-R-09, R-04), and Zhejiang Sci-Tech University Grant 0616033 to Prof Xin-yuan LIU.

\section{Author contribution}

Yi-gang WANG designed research; Yu-mei WU performed research; Xue-tian YUE, Yi YANG, Gong-chu LI and $\mathrm{Na}$ LI contributed new analytical tools and reagents; Kangjian ZHANG and Yi-qiang WANG analyzed data; Yu-mei WU and Yi-gang WANG wrote the paper.

\section{References}

1 Jiang H, Lin JJ, Su ZZ, Goldstein NI, Fisher PB. Subtraction hybridization identifies a novel melanoma differentiation associated gene, mda-7, modulated during human melanoma differentiation, growth and progression. Oncogene 1995; 11: 2477-86.

2 Lebedeva IV, Su ZZ, Chang Y, Kitada S, Reed JC, Fisher PB. The cancer growth suppressing gene mda-7 induces apoptosis selectively in human melanoma cells. Oncogene 2002; 21: 708 18.

3 Su Z, Lebedeva IV, Gopalkrishnan RV, Goldstein NI, Stein CA, Reed JC, et al. A combinatorial approach for selectively inducing programmed cell death in human pancreatic cancer cells. Proc 
Natl Acad Sci USA 2001; 98: 10332-7.

4 Fisher PB, Gopalkrishnan RV, Chada S, Ramesh R, Grimm EA, Rosenfeld MR, et al. mda-7/IL-24, a novel cancer selective apoptosis inducing cytokine gene: from the laboratory into the clinic. Cancer Biol Ther 2003; 2: S23-S37.

5 Chada S, Mhashilkar AM, Liu Y, Nishikawa T, Bocangel D, Zheng $\mathrm{M}$, et al. mda-7 gene transfer sensitizes breast carcinoma cells to chemotherapy, biologic therapies and radiotherapy: correlation with expression of bcl-2 family members. Cancer Gene Ther 2006; 13: 490-502.

6 Miyahara R, Banerjee S, Kawano K, Efferson C, Tsuda N, Miyahara $\mathrm{Y}$, et al. Melanoma differentiation-associated gene-7 (mda-7)/ interleukin (IL)-24 induces anticancer immunity in a syngeneic murine model. Cancer Gene Ther 2006; 13: 753-61.

7 Nishikawa T, Ramesh R, Munshi A, Chada S, Meyn RE. Adenovirus-mediated mda-7 (IL24) gene therapy suppresses angiogenesis and sensitizes NSCLC xenograft tumors to radiation. Mol Ther 2004; 9: 818-28

8 Chada S, Nemunaitis J, Tong A, Zhang Y, Su D, Mhashilkar A, et al. A Phase I dose-escalation study of Ad-mda7 (INGN 241) in patients with advanced carcinoma. Cancer Gene Ther 2001; 8 (Suppl 2): S3.

9 Chada S, Cunningham C, Zhang Y, Su D, Mhashilkar A, Ekmekcioglu S, et al. INGN 241 (Ad-mda-7) induces widespread apoptosis and activates the immune system in patients with advanced cancer. Mol Ther 2003; 7: S446.

10 Zhang ZL, Zou WG, Luo CX, Li BH, Wang JH, Sun LY, et al. An armed oncolytic adenovirus system, ZD55-gene, demonstrating potent antitumoral efficacy. Cell Res 2003; 13: 481-99.

11 Liu XY, Qiu SB, Zou WG, Pei ZF, Gu JF, Luo CX, et al. Effective gene-virotherapy for complete eradication of tumor mediated by the combination of htrail (tnfsf10) and plasminogen k5. Mol Therapy 2005; 11: 531-41.

12 Zhao L, Gu J, Dong A, Zhang Y, Zhong L, He L, et al. Potent antitumor activity of oncolytic adenovirus expressing mda-7/IL24 for colorectal cancer. Hum Gene Ther 2005; 16: 845-58.

13 Kelland L. The resurgence of platinum-based cancer chemotherapy. Nat Rev Cancer 2007; 7: 573-84.

14 Rosenberg B, Vancamp L, Krigas T. Inhibition of cell division in Escherichia coli by electrolysis products from a platinum electrode. Nature 1965; 205: 698-9.

15 Morton RP, Rugman F, Dorman EB, Stoney PJ, Wilson JA, McCormick $\mathrm{M}$, et al Cisplatinum and bleomycin for advanced or recurrent squamous cell carcinoma of the head and neck: a randomized factorial phase III controlled trial. Cancer Chemother Pharmacol 1985; 15: 283-9.
16 Williams SD, Birch R, Einhorn LH, Irwin L, Greco FA, Loehrer PJ. Treatment of disseminated germ-cell tumors with cisplatin, bleomycin and either vinblastine or etoposide. N Engl J Med 1987; 316: 1435-40.

17 Pan QW, Liu BS, Liu J, Cai R, Wang YG, Qian C. Synergistic induction of tumor cell death by combining cisplatin with an oncolytic adenovirus carrying TRAIL. Mol Cell Biochem 2007; 304: 315-23.

18 Pan QW, Zhong SY, Liu BS, Liu J, Cai R, Wang YG, et al. Enhanced sensitivity of hepatocellular carcinoma cells to chemotherapy with a Smac-armed oncolytic adenovirus. Acta Pharmacol Sin 2007; 28: 1996-2004.

19 Xiong J, Peng ZL, Tan X. Effects of adenoviral-mediated mda-7/ IL-24 gene infection on the growth and drug-resistance of drugresistant ovarian cancer cell lines. Sichuan Da Xue Xue Bao Yi Xue Ban 2007; 38: 433-6. Chinese.

20 Khuri FR, Nemunaitis J, Ganly I, Arseneau J, Tannock IF, Romel $\mathrm{L}$, et al. A controlled trial of ONYX-015, a replication-selective adenovirus, in combination with cisplatin and 5-FU in patients with recurrent head and neck cancer. Nat Med 2000; 6: 879-85.

21 Huang EY, Madireddi MT, Gopalkrishnan RV, Leszczyniecka $\mathrm{M}, \mathrm{Su} \mathrm{Z}$, Lebedeva IV, et al. Genomic structure, chromosomal localization and expression profile of a novel melanoma differentiation associated (mda-7) gene with cancer specific growth suppressing and apoptosis inducing properties. Oncogene 2001; 20: 7051-63.

22 Liu XY, Gu JF. Targeting gene-virotherapy of cancer. Cell Res 2006; 16: 25-30.

23 Hajra KM, Tan L, Liu JR. Defective apoptosis underlies chemoresistance in ovarian cancer. Adv Exp Med Biol 2008; 622: 197208.

24 Uyama N, Hatano E, Maetani Y, Isoda H, Shibata T, Taura K, et al. Efficacy and toxicity of transcatheter arterial chemoembolization with cisplatin suspended in lipiodol for unresectable hepatocellular carcinoma. Gan To Kagaku Ryoho 2008; 35: 775-80.

25 Kirn D. Clinical research results with dl1520 (Onyx-015), a replication-selective adenovirus for the treatment of cancer: what have we learned? Gene Ther 2001; 8: 89-98.

26 Kirn D. Oncolytic virotherapy for cancer with the adenovirus d11520 (Onyx-015): results of phase I and II trials. Expert Opin Biol Ther 2001; 1: 525-38.

27 Fillastre JP, Raguenez-Viotte G. Cisplatin nephrotoxicity. Toxicol Lett 1989; 46: 163-75.

28 Arany I, Safirstein RL. Cisplatin nephrotoxicity. Semin Nephrol 2003; 23: 460-4. 